\title{
Should adults take vitamin D supplements to prevent disease?
}

\author{
Mark J Bolland associate professor ${ }^{1}$, Alison Avenell professor ${ }^{2}$, Andrew Grey associate professor ${ }^{1}$ \\ ${ }^{1}$ Department of Medicine, University of Auckland, Auckland 1142, New Zealand; ${ }^{2}$ Health Services Research Unit, University of Aberdeen, Aberdeen
} AB25 2ZD, Scotland

The US Preventive Services Task Force recommends against vitamin $\mathrm{D}$ and calcium supplementation for fracture prevention in otherwise healthy postmenopausal women. ${ }^{1}$ However, despite high quality systematic reviews reporting ineffectiveness, many guideline groups continue to recommend vitamin $\mathrm{D}$ supplementation (with or without calcium) for fall or fracture prevention. Recently Public Health England recommended that everyone needs vitamin $\mathrm{D}$ equivalent to an average daily intake of $10 \mu \mathrm{g}(400 \mathrm{IU})$ to protect bone and muscle health, ${ }^{2}$ and more than $30-50 \%$ of older people in some Western countries take vitamin D supplements. ${ }^{34}$ The role of vitamin D supplementation in individuals not at high risk of osteomalacia (box 1) has been extensively investigated in recent years, but some uncertainties remain.

Since severe vitamin D deficiency causes osteomalacia (box 1), it is reasonable to ask whether less marked reductions in 25-hydroxyvitamin D are associated with musculoskeletal outcomes such as falls and fractures, or surrogate markers such as bone density, muscle function, and parathyroid hormone. Numerous observational studies have shown that low vitamin D status is associated with these musculoskeletal outcomes. These studies must be treated cautiously because observational studies are subject to confounding, and low vitamin D status might be a marker of poor health or lifestyle rather than a causal factor.

Based on these associations, the next step is to ask whether prescribing vitamin D to increase 25-hydroxyvitamin D levels prevents or modifies these outcomes. Although clinical trials show that vitamin D supplementation can lower parathyroid hormone, it is unclear whether this is a valid surrogate for clinical outcomes. Therefore, the effects of vitamin D supplementation must be determined from randomised controlled trials (RCTs) with "hard" clinical outcomes. A large number of RCTs and meta-analyses with clinical outcomes have been done.

Observational and preclinical studies have also associated low vitamin D status with a wide variety of non-skeletal adverse clinical outcomes (box 2) ${ }^{6}$ that are not recognised features of osteomalacia. Trial data and some meta-analyses are now available to understand whether prescribing vitamin $\mathrm{D}$ to increase 25-hydroxyvitamin D makes a difference for such non-musculoskeletal outcomes.

\section{What is the evidence of uncertainty? Musculoskeletal outcomes Vitamin $D$ alone}

Over 50 meta-analyses of vitamin D supplementation and falls or fractures have been published; some report small beneficial effects but others none. These results might seem inconsistent, but the differences are largely explained by differences in methodology. When all available RCTs are included, and all participants from all the studies are analysed by intention-to-treat (rather than "per protocol" or "completers" analyses), there is no consistent evidence that vitamin D supplementation or raising 25-hydroxyvitamin D levels improves musculoskeletal outcomes. ${ }^{8-10}$ In such systematic reviews, vitamin D supplementation (when used as monotherapy without additional calcium supplementation) had no important effects on bone density ${ }^{11}$ nor any consistent effects on falls, ${ }^{12}$ total fracture, or hip fracture (table $1 \Downarrow$ ). ${ }^{13}$ Some individual trials reported statistically significant, clinically relevant increased risks of falls (range of relative risks 1.15-1.40) ) $^{14}$ and fractures (range of relative risks $1.26-1.49)^{14}{ }^{16}$ from intermittent, high dose vitamin D. 
to alter these conclusions. ${ }^{12}{ }^{18}$ Relative risk reductions of $<10 \%$ are unlikely to be attractive to individuals because the absolute benefit of treatment is small, and there is a high likelihood of no benefit from treatment.

Secondly, most of the participants in existing RCTs had baseline 25-hydroxyvitamin D levels of $25-50 \mathrm{nmol} / \mathrm{L}$. If vitamin D supplementation does have benefits, they are most likely to be seen in populations with severe vitamin D deficiency. None of the ongoing trials are targeting these population groups and are therefore unlikely to recruit cohorts with baseline 25 -hydroxyvitamin D levels $<25 \mathrm{nmol} / \mathrm{L}^{5}$

Some earlier trials have reported increased risk of falls or fractures with high vitamin D doses. ${ }^{14-16}$ The ongoing large trials are all using high daily or intermittent dose regimens and should clarify whether such doses are harmful.

\section{What should we do in the light of the uncertainty?}

Osteomalacia is an uncommon but serious illness that can readily be prevented. People at high risk (box 1) should be counselled about sunlight exposure and diet, and low dose vitamin D supplements (400-800 IU/day) can be considered on an individual basis. Otherwise, we conclude that current evidence does not support the use of vitamin D supplementation to prevent disease. This advice is similar to the recommendation of the Scientific Advisory Committee on Nutrition that 25-hydroxyvitamin D of individuals in the UK should not fall below $25 \mathrm{nmol} / \mathrm{L}$. ${ }^{5}$ We believe this can be achieved pragmatically by offering high risk individuals or populations low dose vitamin D of 400-800 IU/day; measurement of 25-hydroxyvitamin D is seldom necessary.

Contributors: MJB drafted the paper. All authors critically reviewed and improved it. MJB is the guarantor for the article. All authors had access to all the data and take responsibility for the integrity of the data and the accuracy of the data analysis.

Competing interests: We have read and understood BMJ policy on declaration of interests and declare: AG is a shareholder in Auckland Bone Density, an organisation that provides bone densitometry services. All authors have published randomised controlled trials and systematic reviews in the fields of vitamin $D$ and calcium.

Funding: Funded by the Health Research Council (HRC) of New Zealand. The authors are independent of the HRC. The HRC had no role in study design, the collection, analysis, and interpretation of data, the writing of the article, or the decision to submit it for publication. The Health Services Research Unit is funded by the Chief Scientist Office of the Scottish Government Health and Social Care Directorate.

Provenance and peer review: Commission; externally peer reviewed.

1 Moyer VA. U.S. Preventive Services Task Force. Vitamin D and calcium supplementation to prevent fractures in adults: U.S. Preventive Services Task Force recommendation statement. Ann Intern Med 2013;158:691-6. doi:10.7326/0003-4819-158-6-20130319000588 pmid:23440163.

2 Public Health England.PHE publishes new advice on vitamin D. 2016. www.gov.uk government/news/phe-publishes-new-advice-on-vitamin-d

3 Bailey RL, Dodd KW, Goldman JA, et al. Estimation of total usual calcium and vitamin D intakes in the United States. J Nutr 2010;140:817-22. doi:10.3945/jn.109.118539 pmid: 20181782.

4 Castro-Lionard K, Dargent-Molina P, Fermanian C, Gonthier R, Cassou B. Use of calcium supplements, vitamin D supplements and specific osteoporosis drugs among French women aged 75-85 years: patterns of use and associated factors. Drugs Aging 2013;30:1029-38. doi:10.1007/s40266-013-0121-9 pmid:24114665.

5 Scientific Advisory Committee on Nutrition (SACN). Vitamin D and Health. 2016. www. gov.uk/government/uploads/system/uploads/attachment_data/file/537616/SACN_Vitamin D_and_Health_report.pdf.

6 Autier $\mathrm{P}$, Boniol M, Pizot C, Mullie P. Vitamin D status and ill health: a systematic review. Lancet Diabetes Endocrinol 2014;2:76-89. doi:10.1016/S2213-8587(13)70165-7 pmid: 24622671.

7 Theodoratou E, Tzoulaki I, Zgaga L, loannidis JP. Vitamin D and multiple health outcomes: umbrella review of systematic reviews and meta-analyses of observational studies and randomised trials. BMJ 2014:348:g2035, doi:10.1136/bmj.g2035 pmid:24690624.

8 Abrahamsen B, Avenell A, Bolland M, et al. A pooled analysis of Vitamin D dose requirements for fracture prevention. IBMS boneKEy 2013;10. doi:10.1038/bonekey.2012. 256.

9 Bolland MJ, Grey A. A case study of discordant overlapping meta-analyses: vitamin d supplements and fracture. PLoS One 2014;9:e115934. doi:10.1371/journal.pone. 0115934 pmid:25551377.

10 Bolland MJ, Grey A, Reid IR. Differences in overlapping meta-analyses of vitamin D supplements and falls. J Clin Endocrinol Metab 2014;99:4265-72. doi:10.1210/jc.20142562 pmid:25093621.

11 Reid IR, Bolland MJ, Grey A. Effects of vitamin D supplements on bone mineral density: a systematic review and meta-analysis. Lancet 2014;383:146-55. doi:10.1016/S01406736(13)61647-5 pmid:24119980.

12 Bolland MJ, Grey A, Gamble GD, Reid IR. Vitamin D supplementation and falls: a trial sequential meta-analysis. Lancet Diabetes Endocrinol 2014;2:573-80. doi:10.1016/S22138587(14)70068-3 pmid:24768505.

13 Avenell A, Mak JC, O'Connell D. Vitamin D and vitamin D analogues for preventing fractures in post-menopausal women and older men. Cochrane Database Syst Rev 2014;4:CD000227.pmid:24729336.

14 Sanders KM, Stuart AL, Williamson EJ, et al. Annual high-dose oral vitamin D and falls and fractures in older women: a randomized controlled trial. JAMA 2010;303:1815-22. doi:10.1001/jama.2010.594 pmid:20460620.

15 Bischoff-Ferrari HA, Dawson-Hughes B, Orav EJ, et al. Monthly high-dose vitamin D treatment for the prevention of functional decline: a randomized clinical trial. JAMA Intern Med 2016;176:175-83. doi:10.1001/jamainternmed.2015.7148 pmid:26747333.

16 Smith H, Anderson F, Raphael H, Maslin P, Crozier S, Cooper C. Effect of annual intramuscular vitamin $\mathrm{D}$ on fracture risk in elderly men and women--a population-based randomized, double-blind, placebo-controlled trial. Rheumatology (Oxford) 2007;46:1852-7. doi:10.1093/rheumatology/kem240 pmid:17998225.

17 Bolland MJ, Leung W, Tai V, et al. Calcium intake and risk of fracture: systematic review. BMJ 2015:351:h4580, doi:10.1136/bmi.h4580 pmid:26420387.

18 Bolland MJ, Grey A, Gamble GD, Reid IR. The effect of vitamin D supplementation on skeletal, vascular, or cancer outcomes: a trial sequential meta-analysis. Lancet Diabetes Endocrinol 2014;2:307-20. doi:10.1016/S2213-8587(13)70212-2 pmid:24703049.

Published by the BMJ Publishing Group Limited. For permission to use (where not already granted under a licence) please go to http://group.bmj.com/group/rights-licensing/ permissions 


\section{Search strategies and trial registries searched}

Our article is based on repeated searches carried out independently by two authors (MJB and AA) to inform systematic reviews of vitamin $D$ published over several years with outcomes of fracture, falls, mortality, cardiovascular disease, stroke, cancer, and adverse events. The full text of the searches are available in the primary references, ${ }^{12-18}$ but we have repeatedly searched Medline, PubMed, Embase, and the Cochrane Library and hand searched reference lists and relevant conference abstracts for randomised controlled trials and systematic reviews of vitamin D in adults. Our most recent search was in December 2015 to identify all published randomised controlled trials of vitamin D supplementation. We also searched ClinicalTrials.gov (https://clinicaltrials.gov/), the International Standard Randomised Controlled Trial Number (ISRCTN) registry (www.isrctn.com/), and the Australian New Zealand Clinical Trials Registry (ANZCTR) (www.anzctr.org.au/) for completed and ongoing trials, using vitamin $\mathrm{D}$ as the search term.

\section{Recommendations for future research}

- Future randomised controlled trials of vitamin $D$ supplementation should focus on populations with severe vitamin $D$ deficiency by enrolling individuals with 25 -hydroxyvitamin $D$ concentrations $<25 \mathrm{nmol} / \mathrm{L}$

- Such individuals would need to be at low risk of osteomalacia at baseline, and the trial protocol would need specific provision for monitoring for osteomalacia. However, there will be costs from identifying sufficient numbers of participants with low 25-hydroxyvitamin D

\section{Education into practice}

- If a middle aged patient who is otherwise well asks you whether they should take vitamin D what would you discuss with them to come to a decision?

- If you saw a housebound older person, how would you consider and discuss the pros and cons of vitamin D with them?

- Based on reading this article is there anything that you would do differently in your practice?

How patients were involved in the production of this article

No patients were involved in the production of this article

\section{Tables}

Table 1| Recent meta-analyses of vitamin D monotherapy which show no statistically significant difference on musculoskeletal outcomes

\section{Outcome No of trials No of participants Relative risk (95\% Cl)}

\begin{tabular}{llll} 
Falls $^{12}$ & 16 & 22291 & $0.95(0.89$ to 1.02$)$ \\
\hline Total fracture $^{13}$ & 15 & 28271 & $1.03(0.96$ to 1.11$)$ \\
\hline Hip fracture $^{13}$ & 11 & 27693 & $1.12(0.98$ to 1.29$)$ \\
\hline
\end{tabular}


Table 2| Recent wide-ranging systematic reviews and Cochrane reviews of randomised controlled trials (RCTs) of vitamin D supplementation with non-skeletal outcomes

\begin{tabular}{|c|c|c|c|}
\hline Review & Outcome & Description & Findings \\
\hline \multicolumn{4}{|c|}{ Comprehensive, large systematic reviews } \\
\hline Autier $2014^{6}$ & $\begin{array}{l}\text { Clinical and surrogate outcomes } \\
\text { (including cardiovascular disease, } \\
\text { mortality, cancer incidence, lipids, } \\
\text { glucose metabolism, physical } \\
\text { function) }\end{array}$ & 172 RCTs & $\begin{array}{l}\text { - No effect on disease occurrence. } \\
\text { - Small reduction in all-cause mortality (RR range } 0.93-0.96) \text {. } \\
\text { - Authors state that RCTs of disease reduction are needed to test } \\
\text { whether associations between low vitamin D status and ill health are } \\
\text { mediated by inflammation. }\end{array}$ \\
\hline Bolland $2014^{18}$ & $\begin{array}{l}\text { Stroke, myocardial infarction, } \\
\text { cancer, fractures, mortality }\end{array}$ & $\begin{array}{l}\text { Trial sequential analysis } \\
\text { of RCTs }\end{array}$ & $\begin{array}{l}\text { - Does not reduce skeletal or non-skeletal outcomes by more than } \\
15 \% \text { in unselected, community dwelling individuals. }\end{array}$ \\
\hline Theodoratou $2014^{7}$ & Clinical and surrogate outcomes & $\begin{array}{l}87 \text { meta-analyses of } \\
\text { RCTs }\end{array}$ & - No consistent effects on health outcomes. \\
\hline \multicolumn{4}{|l|}{ Recent Cochrane reviews } \\
\hline \multirow[t]{2}{*}{ Bjelakovic 2014, (CD007469) } & Cancer & $18 \mathrm{RCTs}$ & • No effect on cancer incidence. \\
\hline & & & $\begin{array}{l}\text { - Reduced cancer mortality in } 4 \text { trials (RR } 0.88(95 \% \mathrm{Cl} 0.78 \text { to } 0.98) \text { ), } \\
\text { but authors rated this low quality evidence }\end{array}$ \\
\hline \multirow[t]{3}{*}{ Bjelakovic 2014, (CD007470) } & Mortality & $56 \mathrm{RCTs}$ & - Reduced mortality by small amount (RR 0.97 ( $95 \% \mathrm{Cl} 0.94$ to 0.99$)$ ). \\
\hline & & & $\begin{array}{l}\text { - Benefit in trials of vitamin D3 (RR } 0.94 \text { (0.91 to } 0.98) \text { ) but not vitamin } \\
\text { D2 (RR } 1.02(0.96 \text { to } 1.08) \text { ). }\end{array}$ \\
\hline & & & $\begin{array}{l}\text { - Authors state that risks of attrition bias, outcome reporting bias, and } \\
\text { other weaknesses warrant further placebo-controlled RCTs }\end{array}$ \\
\hline Ferguson 2014, (CD007298) & Cystic fibrosis & $3 \mathrm{RCTs}$ & - Insufficient evidence to draw reliable conclusions \\
\hline Straube 2015, (CD007771) & Chronic pain & $10 \mathrm{RCTs}$ & $\begin{array}{l}\text { - Insufficient evidence to draw reliable conclusions but large effect } \\
\text { unlikely }\end{array}$ \\
\hline De-Regil 2016, (CD008873) & Pregnancy and newborn outcomes & 15 RCTs & - Insufficient evidence to draw reliable conclusions \\
\hline \multirow[t]{3}{*}{ Martineau 2016, (CD011511) } & Asthma & 7 RCTs (2 in adults) & $\begin{array}{l}\text { - In each trial, vitamin D had no effect on primary or secondary clinical } \\
\text { outcomes. }\end{array}$ \\
\hline & & & $\begin{array}{l}\text { - Reduced rate of exacerbations requiring corticosteroids or hospital } \\
\text { visit. These were not the primary or secondary outcomes. }\end{array}$ \\
\hline & & & $\begin{array}{l}\text { - Authors recommend caution in applying evidence to clinical practice } \\
\text { because results come from few trials. }\end{array}$ \\
\hline
\end{tabular}

$\mathrm{RCT}=$ randomised controlled trial. $\mathrm{RR}=$ relative risk. $\mathrm{Cl}=$ confidence interval. 